\title{
Propranolol en hemangiomas congénitos. Serie de casos
}

\author{
Ricardo Ávila-Reyes*, Rocío Isabel Camacho-Ramírez y Nora Inés Velázquez-Quintana \\ Jefatura de Epidemiología, Unidad de Cuidados Intensivos Neonatales, Hospital Infantil de Tamaulipas, Cd. Victoria, Tamps., México
}

\begin{abstract}
RESUMEN
Introducción: Los hemangiomas infantiles $(\mathrm{HI})$ son los tumores benignos más comunes en la población infantil. En este artículo se reporta el uso del propranolol para el tratamiento de los HI. Casos clínicos: Cuatro neonatos con diagnóstico de hemangioma congénito superficial y aislado fueron tratados tempranamente con propranolol, el cual se empleó en dosis de $2 \mathrm{mg} / \mathrm{kg} /$ día, repartido en dos veces al día, por seis semanas. La resolución de los hemangiomas fue satisfactoria en todos los casos. Discusión: El propranolol es muy efectivo en el tratamiento de los hemangiomas. Se plantea su empleo como primera línea de tratamiento.
\end{abstract}

Palabras clave: Hemangioma congénito. Recién nacido. Propranolol.

\section{ABSTRACT}

Background: Congenital hemangiomas are common benign tumors that affect children. The use of propranolol is reported for the treatment of this disorder. Clinical cases: Four newborns with superficial and isolated congenital hemangiomas, were treated early with propranolol. Propranolol was indicated at a dose of $2 \mathrm{mg} / \mathrm{kg} / \mathrm{day}$ divided twice a day for 6 weeks. The resolution of the hemangiomas was satisfactory in all cases. Discussion: Propranolol is very effective in the treatment of hemangiomas and has been proposed as the first line of treatment. (HOSP MED CLIN MANAG. 2018;11:143-7) Corresponding author: Ricardo Ávila-Reyes, avilareyes@ hotmail.com

Key word: Congenital hemangiomas. New born. Propranolol.

\section{INTRODUCCIÓN}

Los hemangiomas de la infancia $(\mathrm{HI})$ son tumores vasculares benignos diferentes de otros tumores vasculares; de hecho, son los tumores benignos más frecuentes en la infancia. La incidencia va del 5 al 10\% en menores de un año y predominantemente se producen en el sexo femenino ${ }^{1}$.

Su presentación es heterogénea, con variabilidad en distribución, localización y profundidad, según la etapa evolutiva en que se encuentre, y se distribuyen de

\section{Correspondencia:}

*Ricardo Ávila-Reyes

Hospital Infantil de Tamaulipas

Calzada Luis Caballero y Av. Del Maestro, s/n

C.P. 87060 , Cd. Victoria, Tamps.

E-mail: avilareyes@hotmail.com

Date of reception: 09-05-2018

Date of acceptance: 13-09-2018

Doi: 10.24875/HMCM.18000126 
manera segmentaria siguiendo los dermatomas o de manera focal. Respecto a la profundidad, pueden comprometer la dermis (hemangioma superficial), el tejido celular subcutáneo (hemangiomas profundos) o ambos (mixtos). Su localización es mayor en cabeza y cuello $(60 \%)$, tronco $(25 \%)$ y extremidades $(15 \%)$, y en el $80 \%$ de los casos se presentan como lesiones únicas. Las complicaciones pueden presentarse en el $20 \%$ de los pacientes, mayormente en las lesiones segmentarias, siendo la ulceración, compresión u obstrucción de estructuras vecinas las más frecuentes, dependiendo de su localización y tamaño. El diagnóstico de los HI es clínico, y para los casos dudosos de afectación visceral, específicamente en el hígado, se realizan estudios de imágenes, así como en los casos de hemangiomatosis neonatal difusa. La ultrasonografía Doppler y la resonancia magnética son los estudios más precisos ${ }^{1,2}$.

La mayoría de los $\mathrm{HI}$, por su localización, no requieren tratamiento específico debido al curso benigno y autolimitado; sin embargo, el 10-15\% de los casos ameritan tratamiento en la fase proliferativa por el compromiso que puede darse en las estructuras relevantes, como vía aérea, perioculares y hepáticas. La ulceración puede presentarse con dolor intenso y riesgo elevado de infectarse, y los $\mathrm{HI}$ en zonas de fricción incluso pueden producir alteraciones estéticas de relevancia.

El manejo de algunos $\mathrm{HI}$ se realiza con corticoides sistémicos a altas dosis por tiempo prolongado, con las consecuentes complicaciones derivadas de sus efectos adversos, especialmente los hemangioendoteliomas hepáticos infantiles, con éxito comprobado a base de prednisona ${ }^{3}$. Otros manejos incluyen corticoides intralesionales, vincristina, imiquimod, interferón $\alpha$, ciclofosfamida y láser pulsado ${ }^{4}$.

El propranolol es un b-bloqueante no selectivo, descubierto accidentalmente por Leaute-Labreze, et al., que se usa para remitir los hemangiomas ${ }^{5}$. Se han realizado estudios de cohorte y grandes series de casos, y se ha demostrado su utilidad en el manejo de los $\mathrm{HI}$, teniendo respuesta exitosa al tratamiento con propranolol el 95-97\% de los casos.

A continuación, se presentan una serie de casos clínicos de $\mathrm{HI}$ detectados en la etapa neonatal y cuyo tratamiento empezó tan pronto como se realizó el diagnóstico. En ellos se evidencia la respuesta al manejo con propranolol.

\section{CASOS CLÍNICOS}

\section{Caso 1}

Recién nacida de 25 días de vida atendida en la consulta externa por presentar en la región frontal del lado derecho una tumoración de aproximadamente $3 \times 4 \mathrm{~cm}$, prominente, adherida a planos superficiales, movible y tensa a la palpación. La tumoración es diagnosticada como HI congénito, sin involución, localizado y superficial, de acuerdo a la clasificación de hemangiomas². Es decir, no había evidencia de otros hemangiomas similares en su cuerpo. La madre comenta que la niña nació con la tumoración, aunque notó que se hacía más tensa y grande en las siguientes tres semanas de vida. Se decide de forma conjunta iniciar manejo con propranolol a dosis de $2 \mathrm{mg} / \mathrm{kg} / \mathrm{día}$, dividiendo la dosis cada $12 \mathrm{~h}$ por espacio de seis semanas. Es citada para su control a las seis semanas, momento en el cual el $\mathrm{HI}$ presenta menor tamaño en extensión y volumen, y es más suave al tacto, por lo que se deja sin manejo por dos semanas, después de las cuales se reinicia un segundo ciclo de seis semanas de tratamiento con propranolol, ajustando la dosis de acuerdo al peso de la menor. Al finalizar el tratamiento la paciente es evaluada, y se aprecia una mácula de aproximadamente $2 \times 2 \mathrm{~cm}$, sin relieve y con algunas áreas violáceas y rojizas, teniendo involución plena de la masa tumoral (Fig. 1).

\section{Caso 2}

Recién nacido que ingresa en la Sala de Neonatología para el estudio de una tumoración en parrilla costal del lado derecho, a la altura del 6-9 arco costal, línea medio clavicular, de aspecto de rodete, discretamente tenso, prominente y de color rojizo. Se descarta otro tumor similar en el cuerpo, y es diagnosticado como HI localizado, superficial, congénito y que no involuciona, pues se le dio tres semanas para su vigilancia. Al no involucionar, es manejado con propranolol a los 21 días de vida a razón de $2 \mathrm{mg} / \mathrm{kg} / \mathrm{día}$, repartidos en dos veces al día, durante seis semanas. Se le cita a los 15 días después de la culminación del tratamiento, y se aprecia una mácula en forma circular con centro desvanecido, no abultada y sin relieve. Se da seguimiento por varios meses sin recidiva de la tumoración (Fig. 2).

\section{Caso 3}

Recién nacida de 18 días de vida que presenta desde el nacimiento una tumoración en la parrilla costal 


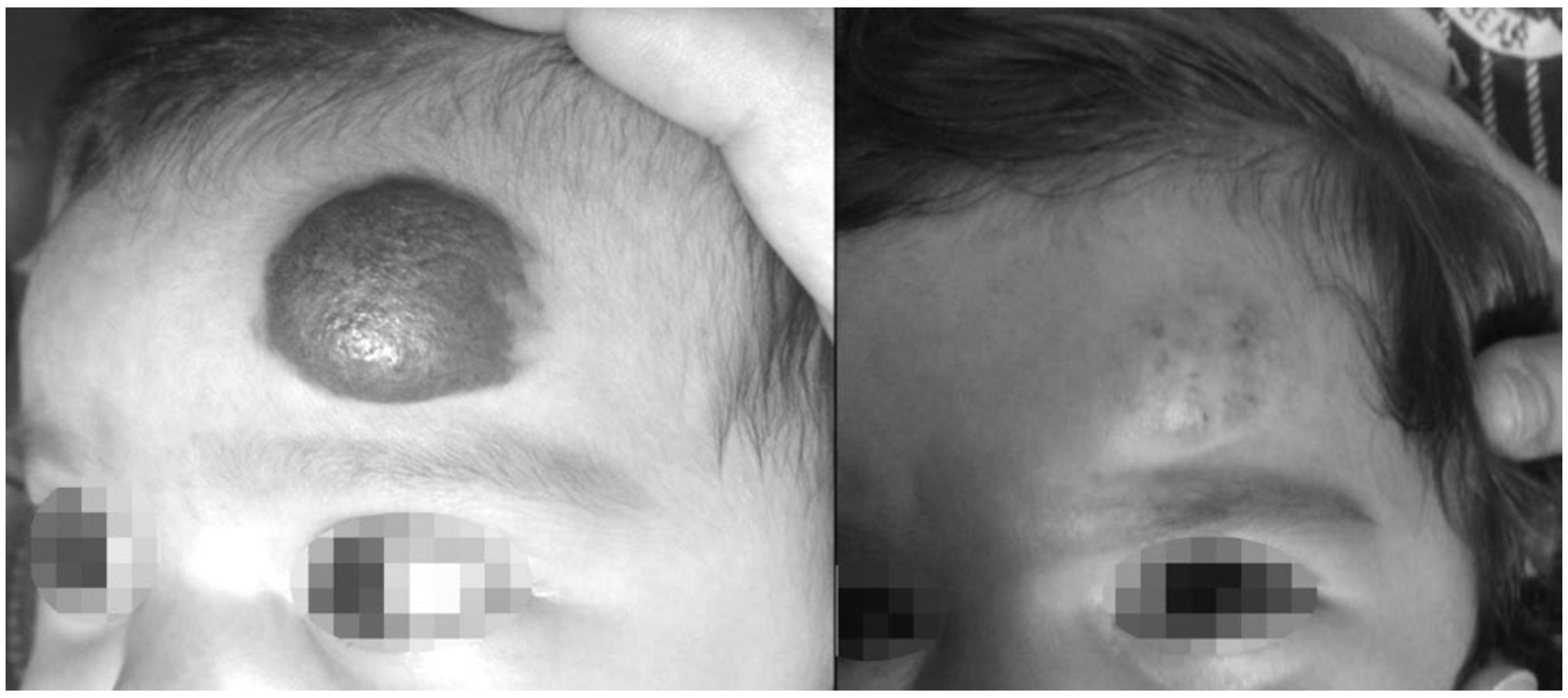

Figura 1. Hemangioma congénito en la frente.

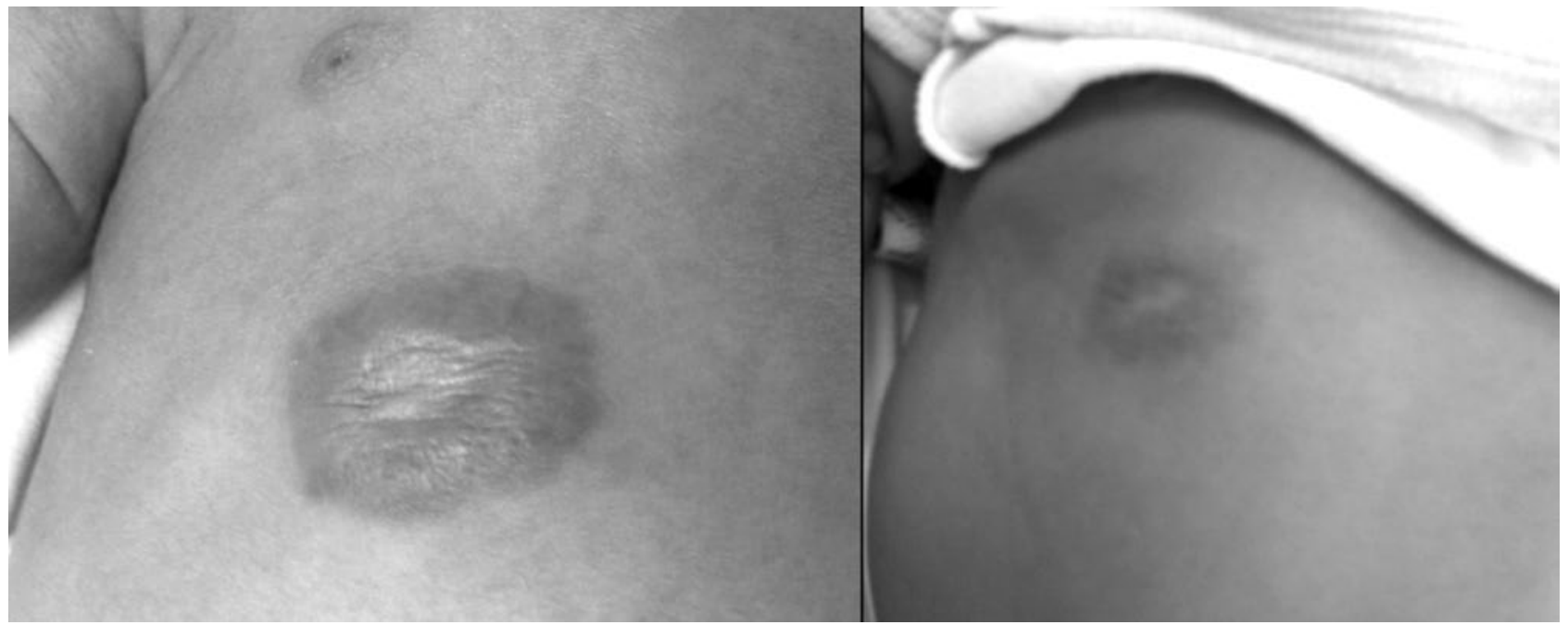

Figura 2. Hemangioma congénito aislado en hemitórax derecho.

derecha, entre línea axilar anterior y línea axilar posterior, desde el sexto arco costal hasta el flanco derecho, de color rojizo, verrugoso a la palpación, superficial y prominente. Es diagnosticado como HI localizado, superficial, congénito y sin involución. Se decide dar manejo con propranolol por seis semanas (2 mg/kg, dos veces al día) teniendo una recidiva del $60 \%$, se deja descansar dos semanas y se vuelve a reiniciar el manejo por dos ciclos más de seis semanas, con 15 días de descanso entre ambas. Se vuelve a ver a la paciente a los ocho meses de edad, y el tumor presenta involución de un 90-95\%, quedando sólo una mácula remanente con zonas rojizas menores y una zona mayormente vascularizada de aproximadamente $1 \times 1 \mathrm{~cm}$, pero no prominente (Fig. 3). Se da seguimiento por dos meses, pero posteriormente se desconoce su evolución por migrar sus padres y la menor a EE.UU.

\section{Caso 4}

Recién nacida de 27 días consultada por presentar desde el nacimiento, sobre la séptima apófisis cervical, una tumoración rojiza de aproximadamente $3 \times 2 \mathrm{~cm}$ sobresaliente, turgente y superficial, diagnosticada clínicamente como $\mathrm{HI}$ localizado, superficial, congénito y que no involuciona debido a la edad de la menor. Se da propranolol a dosis de $2 \mathrm{mg} / \mathrm{kg} /$ día, repartido en dos dosis, en dos ciclos de seis semanas cada uno, con un descanso 


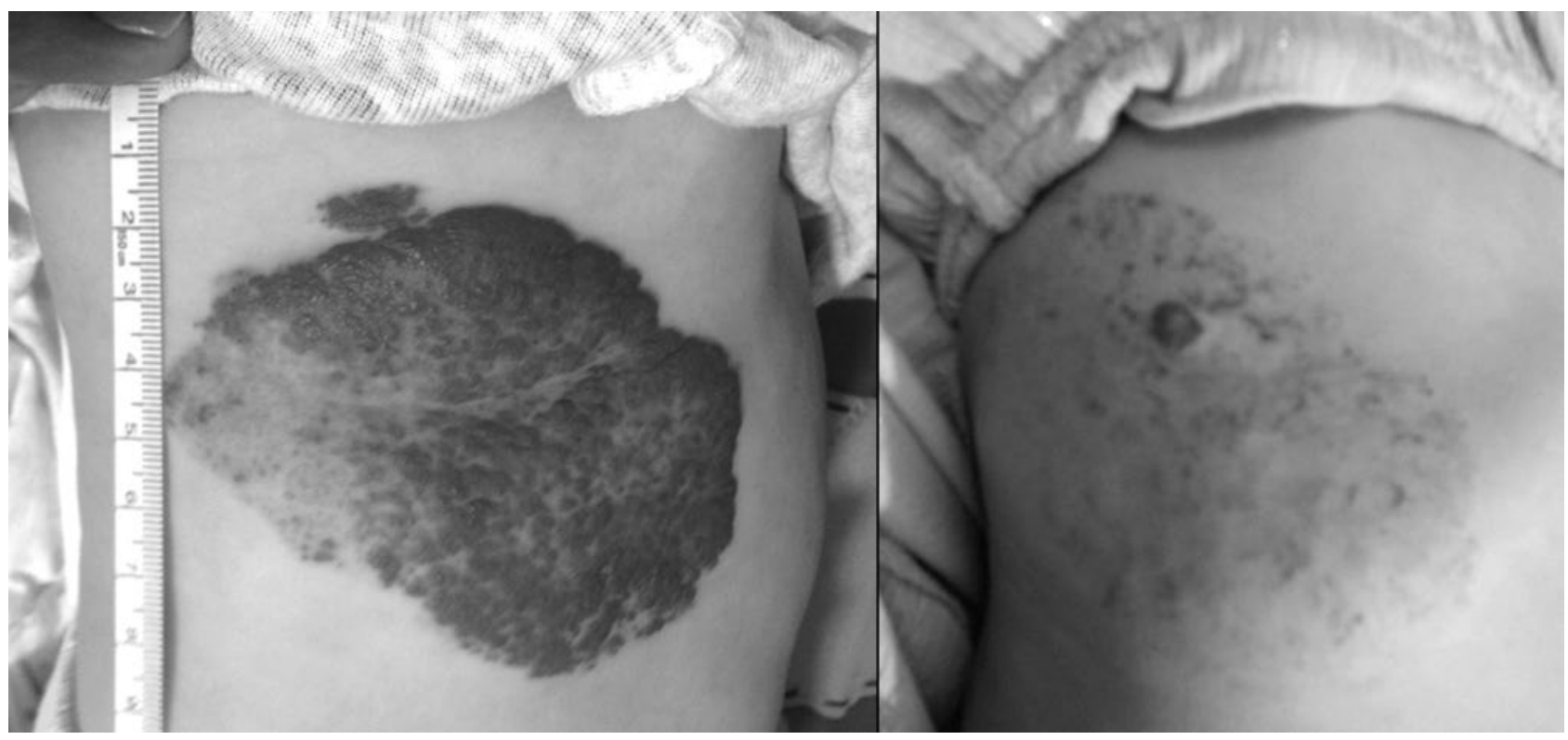

Figura 3. Gran hemangioma congénito en dorso lateral del tórax.

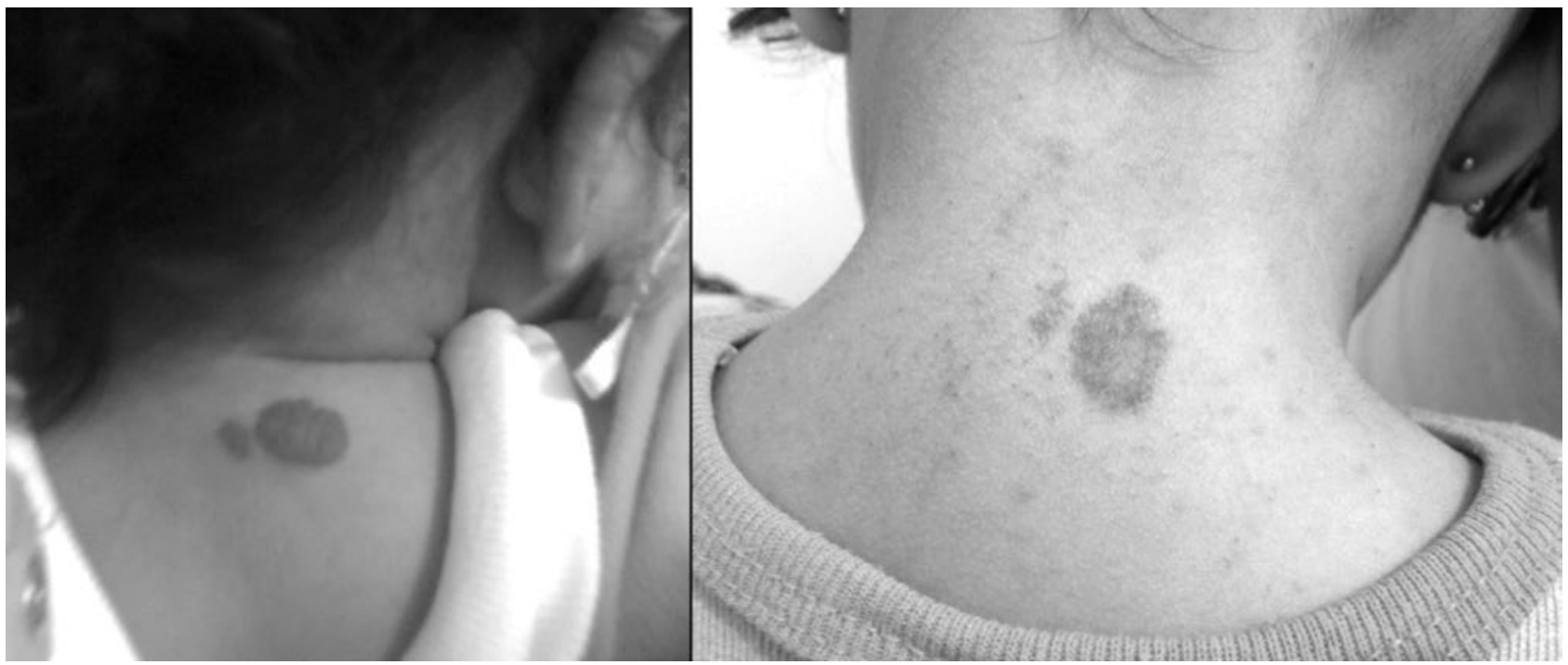

Figura 4. Hemangioma congénito sobre apófisis espinosa de la séptima cervical.

de quince días entre ambos, ajustando la dosis en el segundo ciclo de acuerdo al peso de la menor. Se cita para valoración después del segundo ciclo, pero no regresa hasta el año y seis meses por cambio de ciudad, empero se refiere haber recibido el segundo ciclo por entero. Se revisa y se observa una mácula de $2 \times 2 \mathrm{~cm}$ no sobresaliente de color rojiza y pálida (Fig. 4).

\section{DISCUSIÓN}

El propranolol es un fármaco antagonista no selectivo de los receptores b-adrenérgicos. Su biodisponibilidad oral es cercana al $26 \%$, se elimina por vía hepática casi en su totalidad y su vida media es de 4-5 h. Se emplea primordialmente para tratar enfermedades cardiovasculares como hipertensión arterial, en crisis anóxicas, en la tetralogía de Fallot, taquicardia supraventricular y síndrome de QT largo'. Desde su descubrimiento como terapia para los hemangiomas se han descritos casos aislados y grandes series de tratamiento con elevadas tasas de éxito ${ }^{1,2-10}$. Las características clínicas de los HI difieren. Las lesiones superficiales a veces son pápulas o nódulos rojos, algunas veces lobulados, mientras que las profundas son nódulos suaves de color azulado y pueden tener telangiectasias en la superficie. Los HI 
están dentro del grupo de tumores, y es relevante diferenciar los hemangiomas congénitos, que están formados desde el nacimiento e involucionan rápidamente o no involucionan del todo, por lo que se conocen como hemangiomas congénitos de involución rápida o hemangiomas congénitos que no involucionan. Por tanto, los tumores vasculares se clasifican en hemangiomas, hemangiomatosis, hemangiomas congénitos (involución rápida/que no involucionan), hemangioendotelioma, angioma tufted y granuloma piogénico. Las características clínicas de los HI son variables. Las lesiones superficiales tienden a ser pápulas o nódulos rojos, algunas veces lobulados, mientras las profundas son nódulos suaves de color azulado y pueden tener telangiectasias en la superficie ${ }^{2}$. Los cuatro casos expuestos se diagnosticaron como congénitos, debido a que los pacientes nacieron con la tumoración, y no recidivantes, ya que no involucionaron en las tres primeras semanas, aunque sí se notó un cierto aumento del mismo.

El manejo con propranolol se ha estipulado en dosis bajas, como 2-3 mg/kg/día repartido en cuatro dosis, aunque se han dado casos en que el manejo se ofrece cada $8 \mathrm{~h}$ con buen resultado. Se inicia la dosis a $0.5 \mathrm{mg} /$ kg/día, con la misma dosis de progresión diaria hasta llegar a un máximo de $3 \mathrm{mg} / \mathrm{kg} /$ día cada $8 \mathrm{~h}$. Se recomienda que en menores de tres meses las dosis sean de hasta $2 \mathrm{mg} / \mathrm{kg} / \mathrm{día}$, por el riesgo de hipoglucemia7. Pese a que su indicación preferentemente sea cada $6 \mathrm{~h}$, se sugiere dar cada 8 h. Para tener un mayor apego al tratamiento, incluso se puede llegar a repartir la dosis cada 12 h, como en nuestros casos, en los que decidimos optar por sólo dos dosis al día para que hubiera mayor cumplimiento del tratamiento. Debido a que no se ha estipulado la duración del tratamiento, sólo se considera que debe cubrir toda la fase de crecimiento, se consideró dar seis semanas de manejo, y en el caso de no tener una resolución plena, ofrecer un segundo ciclo por el mismo tiempo dejando dos semanas de intermedio sin manejo para evitar en lo posible las complicaciones al propranolol, que se describen como hipoglucemia, irritabilidad, hipotensión, bradicardia, trastornos del sueño y broncoespasmo. En nuestra breve casuística no tuvimos ningún dato clínico de hipoglucemia ni de ningún otro tipo de efecto adverso descrito en la literatura médica.

Los efectos del propranolol en los hemangiomas se pueden atribuir principalmente a tres mecanismos moleculares: vasoconstricción, inhibición de la angiogénesis e inducción de la apoptosis ${ }^{8}$. La eficacia del propranolol se ha demostrado tanto en $\mathrm{HI}$ como en tumores como hemangioma hepático múltiple, hemangioendoteliomas y hemangioma visceral congénito localizado3,8-10. En algunos casos en los que no suele emplearse propranolol y que suelen manejarse sólo con esteroides e interferón $\alpha$, como hemangioendoteliomas e incluso hemangiomatosis difusa neonatal, ambos con curso fatal ${ }^{8,11}$, se hace énfasis en base a la literatura médica que se debe dar como primera línea de manejo el propranolol para los distintos tumores vasculares, ya que el propranolol se ha demostrado efectivo y accesible, lo que facilita su uso precoz. Las series hasta hoy reportadas apoyan el hecho de que cuanto más temprano sea el tratamiento, más influencia tendrá sobre la fase de crecimiento del $\mathrm{HI}^{4}$, ya que el tratamiento seguramente detendrá el desarrollo y crecimiento que característicamente presenta el $\mathrm{HI}$ durante el primer año de vida; aunque el manejo sea tardío (> 2 años), los resultados son también alentadores.

\section{CONCLUSIONES}

El propranolol resulta efectivo para la reducción del tamaño y extensión de los HI. La dosis indicada en la literatura médica es de 2-3 mg/kg/día, que fue la aplicada en nuestra casuística. En ningún caso hubo efectos adversos al propranolol.

\section{BIBLIOGRAFÍA}

1. Krämer D, Muñoz P, Alfaro P, Araníbar L, Cárdenas L, Morovic CG, et al. Propranolol en el tratamiento de los hemangiomas de la infancia. Rev Chil Pediatr. 2010;81(6):523-30.

2. Balma-Mena A, Lara-Corrales I. Clasificación, manejo y complicaciones de los hemangiomas de la infancia. Acta Pediatr Costarric. 2010;22(1):7-13.

3. Endis-Miranda E, Villamil-Martínez R, Thomas-Olivares PA, Trujillo-Gálvez JJ. Tratamiento exitoso con prednisona en un recién nacido crítico con hemangioendotelioma hepático infantil multifocal sintomático. Arch Argent Pediatr. 2013;111(5):e129-32.

4. González-Díaz MP, Ramos-Gómez LI, Yáñez-Sánchez JM, Marines-Sánchez HM, Páez-Garza JH. Uso sistémico de propranolol en el tratamiento de hemangiomas infantiles. Rev Mex Oftalmol. 2011;84(4):205-14.

5. Léauté-Labrezé C, Dumas-de la Roque E, Hubiche T, Boralevi F. Propranolol for severe hemangiomas of infancy. N Engl J Med. 2008;38(24):2649-51.

6. Handgretinger R. How a accidental discovery paved the way for the treatment of complicated infantile haemangiomas. Acta Pediatr. 2014;103:896-7.

7. Bonini FK, Bellodi FS, Souza EM. Hemangioma infantil tratado com propranolol. An Bras Dermatol. 2011;86(4):763-6.

8. Fernández-Ibieta M, Méndez-Aguirre NA, Girón-Vallejo 0, Ruiz-Jiménez JI. Rápida respuesta de hemangioma hepático multifocal al tratamiento con propranolol. An Pediatr (Barc). 2013;79(2):122-3.

9. Ballbé M, Ferrández MJ, Fuentes MA, Quiles JL, Ardoy F. Hemangioendotelioma hepático difuso de evolución fatal: antes y después del propranolol. Acta Pediatr Esp. 2013;71(11):e376-80.

10. Vanlander A, Decaluwe W, Vandelanotte M, Van Geet C, Cornette L. Propranolol as a novel treatment for congenital visceral haemangioma. Neonatology. 2010;98(3):229-33

11. Ávila-Reyes R, Velázquez-Quintana NI, Herrera-Pen M, López-Garrido E, Camacho-Ramírez RI, Ordóñez-Ánimas J. Hemangiomatosis difusa neonatal. Presentación de un caso. Pediatría de México. 2011;13(1):32-7. 